\title{
Brief Introduction to Atmospheric Cherenkov Radiation and Imaging Observation
}

\author{
Haiyan Yang ${ }^{1, \mathrm{a}}$,Xiaopan $\mathrm{Li}^{1, \mathrm{~b}}$, Guangyang Xu ${ }^{1, \mathrm{c}}$, Hao Kong ${ }^{1, \mathrm{~d}}$, Li Zhou, and Min Shen ${ }^{1, \mathrm{f}}$ \\ ${ }^{1}$ School of Physics and Information Engineering, Zhaotong University, Zhaotong 657000, China; \\ a elaincoco@foxmail.com, bztu_lxp@foxmail.com, c410344843@qq.com, dynkonghao@gmail.com, ${ }^{\text {e}} 398509$ \\ 209@qq.com, ${ }^{\mathrm{f}} 1045439144 @ q q . c o m$
}

Keywords: High energy, Cherenkov radiation, imaging, detection, telescope, $\gamma$ ray

\begin{abstract}
Due to the shielding effect of the Earth's atmosphere, the energy in the ultraviolet band to $10 \mathrm{GeV}$ between the photons before reaching the sea level is almost completely absorbed by the atmosphere, the energy less than $10 \mathrm{GeV} \gamma$-ray can only be detected directly through the high-altitude experiments. As the direct detection of the altitude experiment is limited by the payload, which leads to the limitation of the space detector to detect the $\gamma$-ray in the higher energy area. Therefore, the detection of VHE and UHE $\gamma$ rays can only be detected indirectly by ground experiments, it is mainly through the atmospheric imaging of the Cherenkov telescope (IACT) and the ground extended-air showering array (EAS). This paper mainly introduces the comparison of the atmospheric Cherenkov imaging technology and the more widely used experiments.
\end{abstract}

\section{Atmosphere Cherenkov Radiation}

Unlike the acceleration of charged particles, the Cherenkov radiation is not the radiation effect of a single particle, but rather the collective effect of moving charged particles and the bound charge and induced current in the medium. When a charged particle passes through a transparent medium with a refractive index of $\mathrm{n}$ at a velocity $\mathrm{v}$, if $\mathrm{v}$ is greater than the phase velocity $\mathrm{c} / \mathrm{n}$ of the light in the medium (c is the speed of light in the vacuum), the particle will produce the Cherenkov radiation. When the charged particles pass through the medium, due to the correlation between the electromagnetic radiation, in a certain direction is strengthened, resulting in Cherenkov radiation ${ }^{[1-2]}$. Figure 1 shows the wave front formation schematic for the conical surface of the Cherenkov radiation. The relationship between the direction of radiation and the direction of particle movement is the angle $\theta_{\mathrm{c}}$ of Cherenkov, and the relationship between them is described by the following relation:

$$
\cos \theta_{c}(\lambda)=\frac{c}{n(\lambda)_{V}}=\frac{1}{n(\lambda) \beta}
$$

Thus, it can be seen that the emission angle $\theta c$ of the Cherenkov radiation is related to the velocity of the charged particles. The condition of the Cherenkov radiation is $\mathrm{v}>\mathrm{c} / \mathrm{n}$, i.e., the threshold velocity of the Cherenkov radiation in the medium is $\mathrm{vT}=\mathrm{c} / \mathrm{n}$ and the corresponding Cherenkov threshold is:

$E_{T}=\frac{m_{0} c^{2}}{\sqrt{1-\beta_{T}{ }^{2}}}=\frac{m_{0} c^{2}}{\sqrt{1-\left(\frac{1}{n}\right)^{2}}}$

In which, is the refractive index, and $\mathrm{m} 0$ is the quiet mass of charged particles. The relationship between the air density and the altitude index decreases:

$\rho(h)=\rho_{0} \cdot \exp \left(-\frac{h}{h_{0}}\right)$

It can be known that the relationship between the refractive index varies with altitude:

$n=1+n_{h}=1+n_{0} \cdot \exp \left(-\frac{h}{h_{0}}\right)$ 
In which, $\mathrm{h}_{0}=7.1 \mathrm{~km}$ and $\mathrm{n}_{0}=0.00029$. With the increase of the altitude $\mathrm{h}$, the refractive index decreases, and the threshold of the production of the Cherenkov light increases. Figure 2 shows the curve of the maximum Cherenkov radiation angle with altitude. Figure 3 shows the curve of the Cherenkov 's energy threshold with altitude. It can be seen that at sea level, its maximum Cherenkov radiation angle is 1.38 degrees, the electron's Cherenkov light threshold can be 21.2MeV.

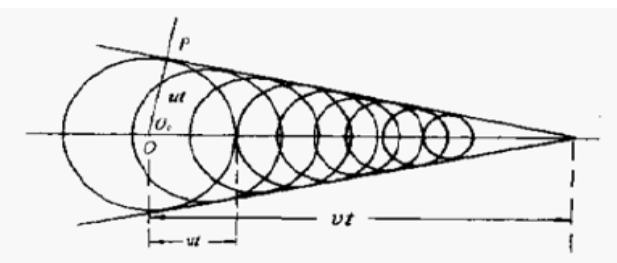

(a)

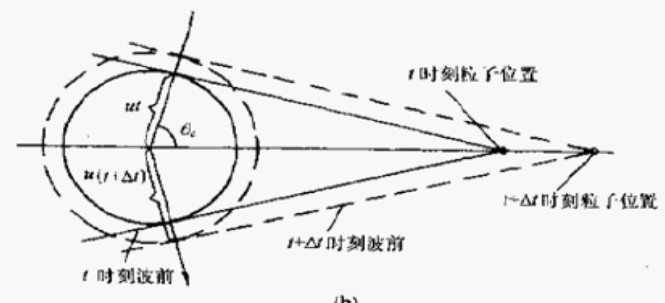

(b)

Fig. 1 Waveform Forming Schematic Diagram of Radial Cone Shape in Cherenkov Radiation
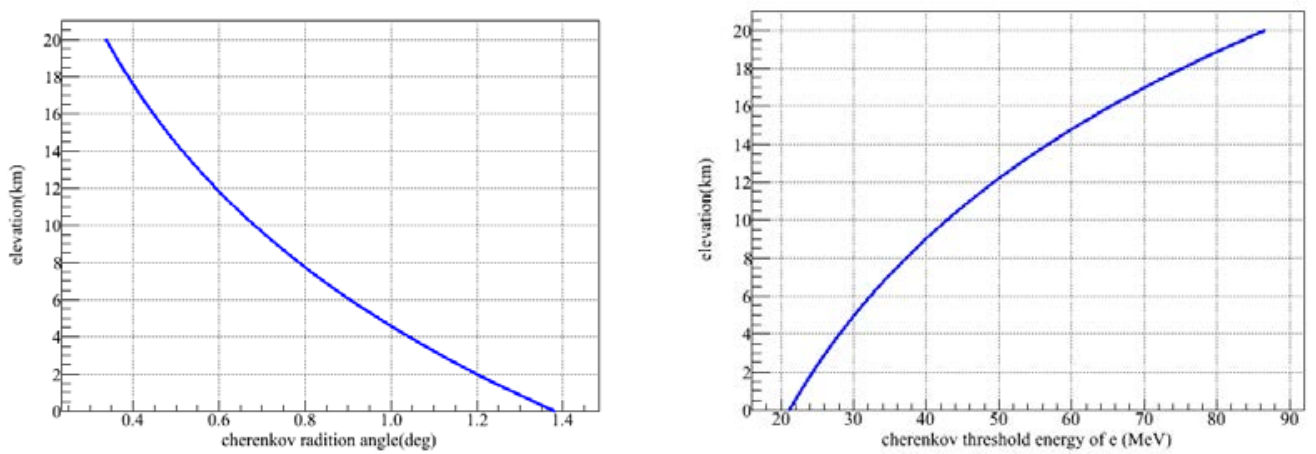

Fig. 2 .(on left)Relationship between the Maximum Radiation Angle of Cherenkov Radiation and the Altitude

Fig. 3.(on right) Curve of the Relationship between the Threshold of Generating Electronic Cherenkov Radiation and the Altitude

\section{Development Features of Atmosphere Cherenkov Radiation}

Atmospheric showering includes electromagnetic cascade shower and hadron cascade shower, different from the original formation of the Cherenkov photon distribution is very different, $\gamma$-ray induced by the electromagnetic cascade process formed by the Cherenkov image More closely (about $0.3^{\circ}$, with energy), and the cosmic line of the strong sub-cascade formed by the Cherenkov like a wide range of expansion, and it is composed by a number of electromagnetic cascade superpositions.

The distribution of the photon number varies with the energy of the original proton, the angle of incidence and the corresponding depth of the atmosphere. For its longitudinal distribution, the Cherenkov light density is lagging behind the development of charged particles, and the particle density is mainly affected by the development of electrons.

For the imaging of the Cherenkov telescope, according to the characteristics of Cherenkov can be judged on the vertical distribution of shower development. The $\gamma$-ray produced by the Cherenkov light is like a dense, asymmetric, and energy-rich hadron produced by the Cherenkov light is essentially isotropic, the resulting image symmetry. $\gamma$-ray produced by the Cherenkov light into the 
spindle type, as shown in Figure 4, the spindle represents the original position ${ }^{[3]}$. Figure 4 shows the image of the Cherenkov light produced by the hadron.
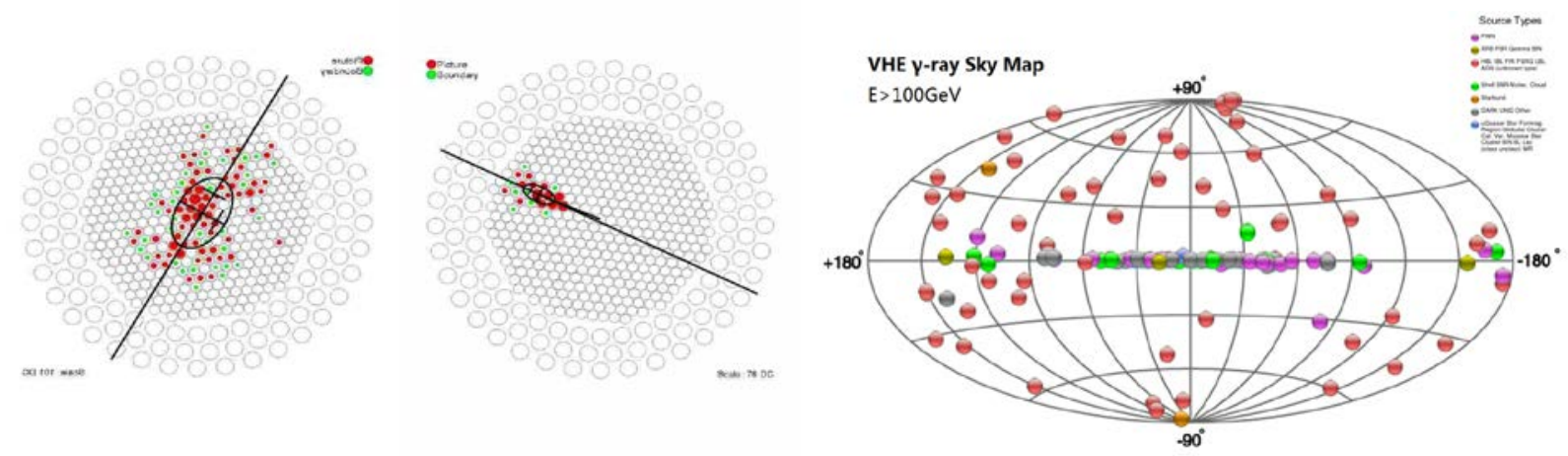

Fig.4. (on left) Image of Cherenkov Light Produced by Hardon and $\gamma$-ray

Fig.5. (on right) The latest $\mathrm{TeV} \gamma$ ray source profile ${ }^{[5]}$

\section{Atmospheric Cherenkov Imaging Telescope}

At present, the $\gamma$-ray ground detection technology mainly includes the atmosphere Cherenkov imaging telescope and EAS array. The atmospheric Cherenkov image imager (IACT) is the charged particles in the atmosphere produced by the Cherenkov light through a lot of mirrors reflected to the corresponding photomultiplier tube, each photomultiplier tube covering a direction area (such as $0.1^{\circ} \times 0.1^{\circ}$ ), so that the extension of the atmosphere can be cascading shower development process presented. Since the Cherenkov light is fixed in the direction of the particle movement, and most of the photons are produced in the vicinity of the shower to the maximum, thus retaining the original cosmic ray information. The energy of the original particles can be reconstructed by the total number of photons collected. The direction of the original particles can be reconstructed by the difference of the Cherenkov image on the telescope, and the distinction between the $\gamma$-ray and the original cosmic ray can be realized.

Although the IACT field of view is small and can only run in the sunny night, but because of its good angular resolution, strong $\gamma$-ray and cosmic background discrimination ability, lower detection threshold, has become Most of the high-energy gamma-ray sources found on the ground have been discovered by IACT, and the number of sources is increasing year by year. Currently running IACT experiments are mainly CANGAROO III, H.E.S.S, MAGIC, VERITAS. Their main performance parameters are shown in Table 1.

Figure 5 shows the $144 \mathrm{TeV} \gamma$ sources detected by the experimental groups in May 2013, the vast majority of which are found by IACT. However, due to the small field of vision of IACT, and only in the sunny night without night to run the environment, the annual observation time is only about $10 \%$. Which limits its search for $\gamma$-source in the whole area. The ground EAS array wide field of view, to all-weather $\gamma$ astronomical observations, it has become an indispensable means of gamma astronomical research. 
Table 1 Main Performance Parameters of IACT $^{[4]}$

\begin{tabular}{|c|c|c|c|c|c|c|c|c|c|}
\hline $\begin{array}{l}\text { Experiment } \\
\text { Name }\end{array}$ & $\begin{array}{l}\text { Latitud } \\
\text { e }\left(^{\circ}\right)\end{array}$ & $\begin{array}{l}\text { Longi } \\
\text { tude ( } \\
\left.{ }^{\circ}\right)\end{array}$ & $\begin{array}{l}\text { Heigh } \\
\text { t (m) }\end{array}$ & $\begin{array}{l}\text { Mirro } \\
\text { r }\end{array}$ & $\begin{array}{l}\text { Area } \\
\left(\mathrm{m}^{2}\right)\end{array}$ & $\begin{array}{l}\text { Total } \\
\text { Area } \\
\left(\mathrm{m}^{2}\right)\end{array}$ & $\begin{array}{l}\text { View } \\
\text { Field } \\
\left(^{\circ}\right)\end{array}$ & $\begin{array}{c}\text { Thresh } \\
\text { old } \\
\text { Energ } \\
\text { y } \\
(\mathrm{TeV})\end{array}$ & $\begin{array}{c}\text { Sensitivit } \\
\mathrm{y} \\
\text { (\%Crab/ } \\
\text { 50hr) }\end{array}$ \\
\hline H.E.S.S. & -23 & 16 & 1800 & 4 & 107 & 428 & 5 & 0.1 & 0.7 \\
\hline VERITAS & 32 & -111 & 1275 & 4 & 106 & 424 & 3.5 & 0.1 & 1 \\
\hline MAGIC & 29 & 18 & 2225 & 1 & 236 & 236 & 3.5 & 0.05 & 1.6 \\
\hline MAGIC-II & & & & 2 & & 472 & & & 0.8 \\
\hline $\begin{array}{c}\text { CANGAROO- } \\
\text { III }\end{array}$ & -31 & 137 & 160 & 3 & 57.3 & 172 & 4 & 0.4 & 15 \\
\hline
\end{tabular}

\section{Reflective Telescopes and Trans-Missive Telescopes}

At present, IACT experiments mainly use large mirrors, the detector placed in the focus of the location, this mirror is difficult to achieve a large field of view, the main reason is the current IACT $\mathrm{f}$ number of 1.2 or so, to get a large field of view, The $\mathrm{f}$ number to be about 2 , this condition is difficult to achieve in the mechanical aspects, and the need for large, expensive camera, but with the camera area will block the mirror itself to accept the light, and thus part of the sacrifice Field of view, as shown in Figure 6 left.
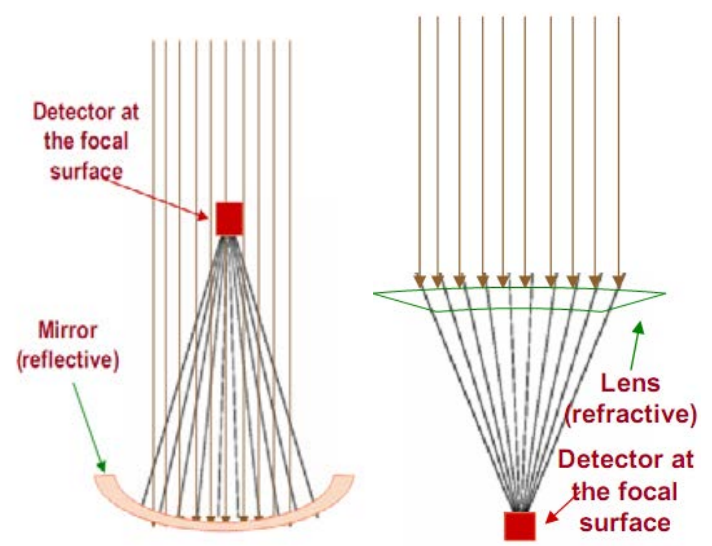

Fig. 6. Mirrors and Transmitters

In order to obtain a large field of view, GAW experiments using Fresnel lens to achieve. Here the Fresnel lens can replace the mirror imaging, but different from the mirror field of view, the light through the lens to reach the camera, the camera size does not affect the light incident on the lens surface, as shown in Figure 6.

French physicist Augustin.Fresnel invented a fine structure of the optical element - Fresnel Lense, Fresnel lens aberration than the traditional optical lens ${ }^{[6]}$, so its image quality worse than the traditional lens.

\section{Conclusion}

At present, for high-energy gamma astronomical observations, ground equipment is divided into two categories, namely, reflective and trans-missive, reflective observation has been a lot of results, 
transmission or a new type of observation, the current only GAW impact Larger, of course, there are many scientists in considering the use of traditional lenses instead of Fresnel lens to try high-energy gamma astronomical observations, and even made a lot of effort. Fresnel lens is more lightweight than traditional optical lens, easy to manufacture, it is rich in manufacturing materials, the cost is relatively low, caliber than the traditional optical lens, the thickness is also very thin, widely used in various industries in many areas ${ }^{[7]}$. However, the imaging quality of Fresnel lenses is generally not as good as that of traditional optical lenses, especially large-diameter Fresnel lenses, for imaging systems where the image quality is relatively high. The lens still cannot replace the traditional optical lens. Theoretically, the traditional optical lens imaging quality is much higher than the Fresnel lens, and has the advantage of large field of view, applied to high-energy gamma astronomical observations is also possible. Want to really achieve this idea, we also need our common efforts.

\section{References}

[1] Xie Yigang et al. Particle Detector and Data Acquisition. Science Press, Beijing,2003: 259.

[2] Chen Lihong. Calibration of Position Response of Photomultiplier Tube and Simulation of $\mu$ Detector. Shijiazhuang: Hebei Normal University, 2011,21-22.

[3] A. M. Hillas. J. Phys, 16(1990), 1271-128.

[4] F. Aharonian et al. Rep. Prog. Phys., 71(2008)

[5] Information on http://www.TeVcat.com.

[6] A. Horibe, M. Baba, E. Nihei, and Y. Koike, "High Efficiency and High Visual Quality LCD Backlighting System", SID, 12(1998), 153.

[7] P.N.Murgatroyd, Theory of space-charge limited current enhancd by frenkel effect, J.Phys.D: AppI.Phys, 3(1970),151. 